'Departamento de Endocrinología, Facultad de Medicina, Pontificia Universidad Católica de Chile. Santiago, Chile. ${ }^{2}$ Programa de Tumores Hipofisarios UC, Red de Salud UC-CHRISTUS. Santiago, Chile.

${ }^{3}$ Centro Traslacional de Endocrinología (CETREN-UC), Red de Salud UCCHRISTUS. Santiago, Chile. ${ }^{4}$ Departamento de Neurocirugía, Facultad de Medicina, Pontificia Universidad Católica de Chile. Santiago, Chile.

${ }^{5}$ Departamento de Radiología, Facultad de Medicina, Pontificia Universidad Católica de Chile. Santiago, Chile. ${ }^{6}$ Departamento de Medicina Intensiva, Facultad de Medicina, Pontificia Universidad Católica de Chile. Santiago, Chile.

Los autores declaran no tener conflictos de interés.

Trabajo no recibió financiamiento.

Recibido el 18 de noviembre de 2019, aceptado el 16 de junio de 2020 .

Correspondencia a: Dra. Flavia Nilo C. Diagonal Paraguay $362,4^{\circ}$ piso, Departamento de Endocrinología, Santiago. ffnilo@uc.cl

Dr. Francisco Guarda. Diagonal Paraguay 362, 4to piso, Departamento de Endocrinología, Santiago. fjguarda@uc.cl

\section{Hipopituitarismo postraumatismo encefalocraneano: revisión de la literatura y algoritmo de estudio y abordaje terapéutico}

\author{
CAROLINA CARMONA R. ${ }^{1}$, PABLO VILLANUEVA G. ${ }^{2,4}$, \\ ISIDRO HUETE ${ }^{2,5}$, JUAN PABLO CRUZ ${ }^{2,5}$, SEBASTIÁN BRAVO ${ }^{6}$, \\ FRANCISCO J. GUARDA V. ${ }^{1,2,3}$, FLAVIA NILO C. ${ }^{1,2,3}$
}

\section{Hypopituitarism after traumatic brain injury}

Hypopituitarism after moderate or severe traumatic brain injury (TBI) is usually underdiagnosed and therefore undertreated. Its course can be divided in an acute phase during the first 14 days after TBI with 50 to $80 \%$ risk of hypopituitarism, and a chronic phase, beginning three months after the event, with a prevalence of hypopituitarism that ranges from 2 to $70 \%$. Its pathophysiology has been addressed in several studies, suggesting that a vascular injury to the pituitary tissue is the most important mechanism during the acute phase, and an autoimmune one during chronic stages. In the acute phase, there are difficulties to correctly interpret pituitary axes. Hence, we propose a simple and cost-effective algorithm to detect and treat a potential hypothalamic-pituitary-adrenal axis impairment and alterations of sodium homeostasis, both of which can be life-threatening. In the chronic phase, post-concussion syndrome is the most important differential diagnosis. Given the high prevalence of hypopituitarism, we suggest that all pituitary axes should be assessed in all patients with moderate to severe TBI, between 3 to 6 months after the event, and then repeated at 12 months after trauma by a specialized team in pituitary disease.

(Rev Med Chile 2020; 148: 1796-1805)

Key words: Brain Injuries, Traumatic; Hypopituitarism; Post-Concussion Syndrome.
11 1 traumatismo encefalocraneano (TEC) es la principal causa de muerte y discapacidad en adultos jóvenes. Se estima una incidencia de 200 por 100.000 habitantes-año, siendo el sexo masculino el más afectado y los accidentes de tránsito la principal causa ${ }^{1}$. El TEC se clasifica en leve, moderado y grave, según la escala de coma de Glasgow (GCS), duración del compromiso de conciencia y amnesia postraumática (Tabla $1)^{2}$. Es importante considerar que los registros están basados en hospitalizaciones, es decir, TEC moderados a graves, por lo que su incidencia está subestimada.

El hipopituitarismo post-TEC (HTEC) es una entidad subdiagnosticada y poco reconocida. Hasta el año 2000 solo había 367 casos descritos en la literatura, pero posteriormente cobró relevancia con la aparición de múltiples estudios de diferente calidad metodológica y protocolos de evaluación endocrina de HTEC, mostrando prevalencias muy disímiles. En el año 2007, Schneider y colaboradores publicaron un metaanálisis de 14 estudios, 
Hipopituitarismo postraumatismo encefalocraneano - C. Carmona et al

Tabla 1. Clasificación de la gravedad del traumatismo encefalocraneano

\begin{tabular}{|lccc|}
\hline Variable & Leve & Moderado & Grave \\
\hline Escala de coma Glasgow & $13-15$ & $9-12$ & $3-8$ \\
\hline Alteración de conciencia & $\leq 24 \mathrm{~h}$ & $>24 \mathrm{~h}$ & $>24 \mathrm{~h}$ \\
\hline Pérdida de conciencia & $<30 \mathrm{~min}$ & $30 \mathrm{~min}$ a $24 \mathrm{~h}$ & $\geq 24 \mathrm{~h}$ \\
Amnesia Postraumática & $\leq 24 \mathrm{~h}$ & $>24 \mathrm{~h} \mathrm{y}<7$ días & $\geq 7$ días \\
\hline
\end{tabular}

encontrando una prevalencia de HTEC en fase crónica de $27,5 \% \%^{3}$.

No existen a nivel nacional estudios de prevalencia ni guías de manejo de HTEC, por lo que realizamos una revisión de la literatura y elaboramos un algoritmo de estudio y abordaje terapéutico que pretendemos sea una guía práctica en la atención integral de nuestros pacientes.

\section{Historia}

El HTEC fue reportado por primera vez en 1918 por Cyran en un paciente con fractura de base de cráneo ${ }^{4}$. Sin embargo, previamente ya se había descrito un cuadro sugerente en Napoleón
Bonaparte (1769-1821), quien sufrió durante su vida múltiples traumatismos, incluyendo un TEC con compromiso de conciencia al caer violentamente de un coche en movimiento, en 1803. Posteriormente, el Barón Fain describió, en 1809, los siguientes cambios en Napoléon: "obesidad, movimientos más lentos, voz menos imperiosa...", y el informe oficial de su autopsia, por Walter Henry Cavan, describió la presencia de "obesidad, alopecía, atrofia de genitales, piel blanca y delicada", signos sugerentes de hipogonadismo, pudiendo constituir un HTEC dado el antecedente de trauma (Figura 1$)^{5}$. Tuvo que pasar casi un siglo para que el HTEC despertara el interés suficiente como para realizar estudios que evaluaran su relevancia ${ }^{6}$.

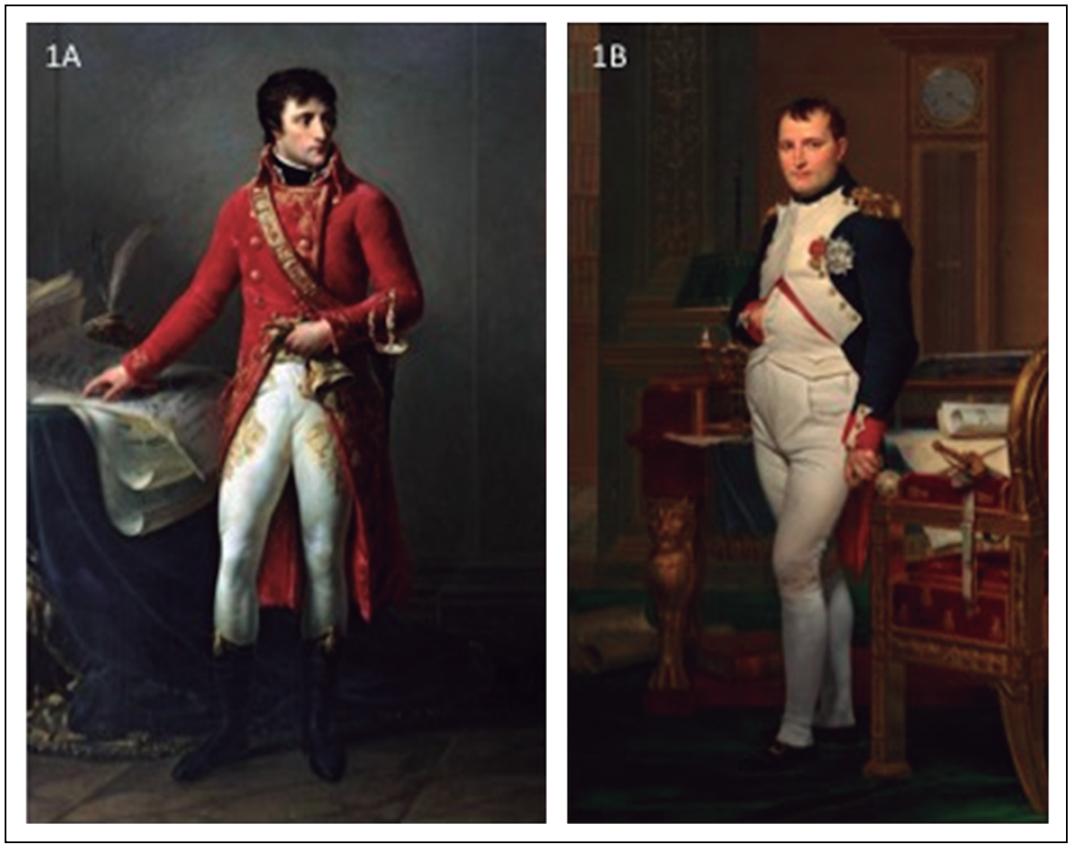

Figura 1. Retratos de Napoleón Bonaparte. A: Antoine-Jean Gros, 1802. B: Jacques-Louis David, 1812. Adaptado de ${ }^{5}$. 


\section{Fisiopatología}

Varios factores han sido implicados en el desarrollo del HTEC ${ }^{7}$, siendo el daño vascular uno de los principales, sustentado en una alta prevalencia de hipopituitarismo posterior a eventos isquémicos (hasta $37,5 \%$ a 15 meses de seguimiento) ${ }^{8}$ y que se ha reconocido daño traumático de los vasos largos que drenan la adenohipófisis con infarto venoso de ella. Estos son los más susceptibles a daño mecánico y explica la mayor prevalencia de compromiso adenohipofisario ${ }^{9,10}$. Además, los estudios post mortem muestran una alta prevalencia de necrosis y hemorragia hipofisarias luego del trauma, sugiriendo un daño traumático directo ${ }^{11}$. Por otra parte, múltiples noxas como hipotensión, hipoxia, anemia e inflamación cerebral pueden favorecer un daño isquémico hipofisario ${ }^{12}$. Finalmente, están descritas secciones traumáticas del tallo y daño al sistema portal, con el consiguiente panhipopituitarismo y diabetes insípida (DI) ${ }^{7,9}$.

Además de los mecanismos descritos, investigaciones recientes han reportado una posible interacción entre autoinmunidad y HTEC, dada la presencia de anticuerpos antihipofisarios y antihipotalámicos que persisten luego de 5 años del trauma y explicarían la aparición tardía de HTEC; sin embargo, faltan mayores estudios para demostrar un rol predictor o patogénico de estos anticuerpos ${ }^{13,14}$.

\section{Historia natural}

Se describe una fase aguda de HTEC que ocurre los primeros 14 días posteriores al traumatismo y una fase crónica desde los 3 meses en adelante ${ }^{15}$. La prevalencia del HTEC en TEC moderados a graves se describe entre 50 y $80 \%$ en la fase aguda y entre 2 y $70 \%$ en fase crónica ${ }^{16}$. Esta variabilidad está influenciada por diversos factores que hacen difícil la interpretación del estudio de ejes hipofisarios en agudo, como el tiempo transcurrido desde el evento, tipo y gravedad del trauma, población estudiada, criterios diagnósticos de hipopituitarismo, entre otros ${ }^{16,17}$.

Pese a esta heterogeneidad, los estudios concuerdan en que los ejes más frecuentemente comprometidos en ambas fases son el somatotropo (30\%) y gonadotropo (25\%), seguidos del corticotropo (20\%) y DI (15\%), siendo estos dos últimos la principal causa de morbimortalidad (Figura 2) ${ }^{6}$.

\section{Evaluación del HTEC en fase aguda y crónica}

\section{Fase aguda}

La evaluación de todos los ejes hipofisarios post-TEC en agudo es considerada una medida poco costo-efectiva, debido a que los cambios fisiopatológicos frente al estrés del traumatismo y shock hacen difícil la interpretación de los resultados $^{18,19}$ :

\section{a) Insuficiencia corticoidal del paciente crítico (CIRCI) versus insuficiencia corticotropa por hipopituitarismo agudo}

En el paciente crítico, durante las primeras 4 semanas se produce un aumento del cortisol que es mayoritariamente no dependiente de ACTH,

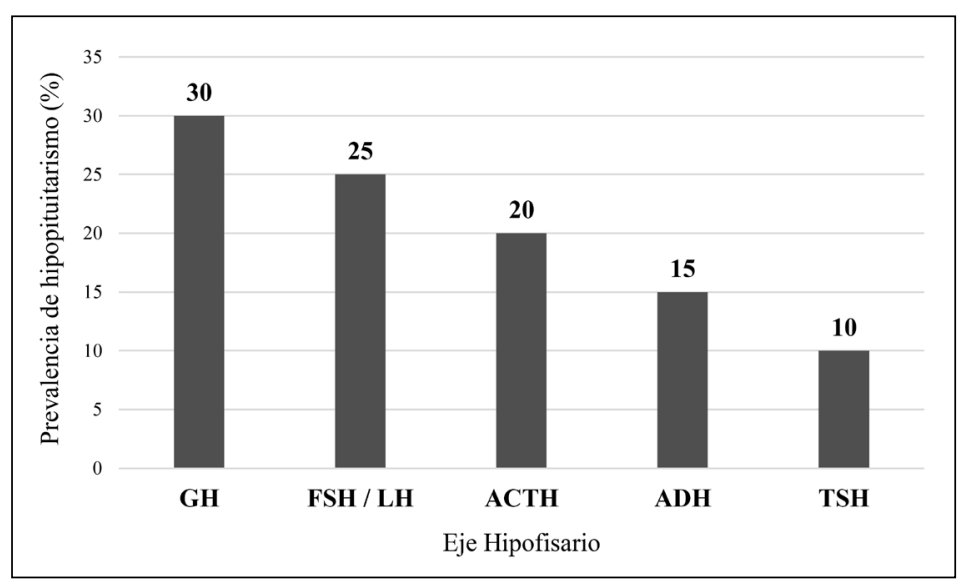

Figura 2. Frecuencia general de HTEC según eje hipofisario comprometido. Adaptado de Quinn $\mathrm{M}^{1}$ (\%). GH: Hormona de crecimiento. LH: Hormona luteinizante. FSH: Hormona folículoestimulante. ACTH: Hormona adrenocorticotrofina. ADH: Hormona antidiurética. TSH: Hormona tiroestimulante. 
asociado a pérdida del ritmo circadiano. Esto se correlaciona con un menor metabolismo hepático y renal, cambios en su volumen distribución por disminución de proteínas transportadoras como albúmina y la globulina fijadora de corticoesteroides y resistencia tejido-específica del receptor de glucocorticoides. Luego de 4 semanas de la noxa, la cortisolemia vuelve a su basal y a depender de ACTH; sin embargo, hay pacientes cuya respuesta a estrés no es óptima, lo que se asocia a niveles insuficientes de cortisol $^{20}$. La incidencia de CIRCI está descrita de 1-6\% en pacientes críticos y hasta $75 \%$ en casos de shock refractario, lo que se ha asociado a mayor dependencia de vasoactivos, falla orgánica múltiple, hipotermia, dificultad en el destete de ventilación mecánica y mortalidad ${ }^{21,22}$. No obstante, no se ha demostrado beneficio en mortalidad con el aporte de hidrocortisona ${ }^{23}$. Lo más utilizado para el diagnóstico de CIRCI es el cortisol sérico, con puntos de corte propuestos $<10 \mathrm{ug} / \mathrm{dL}$ o $<15 \mathrm{ug} / \mathrm{dL}$ para insuficiencia ${ }^{6,17,24,25}$. Si bien el test de estimulación con ACTH 250 ug es considerado el gold standard para confirmar la suficiencia corticotropa, en la fase aguda tanto del HTEC como en CIRCI no tiene utilidad, ya que no ha transcurrido el tiempo suficiente para provocar atrofia suprarrenal y la consiguiente menor respuesta a ACTH exógena. Dado que la técnica para el diagnóstico de ambos es la misma, diferenciarlos es complejo. Considerando que la morbimortalidad de una deficiencia de cortisol por HTEC no tratada es alta, sugerimos iniciar hidrocortisona ante la sospecha clínica de insuficiencia, como presencia de shock refractario, hiponatremia persistente o hipoglicemia, o valores disminuidos de cortisol. Aunque es controvertido un punto de corte en particular, valores $<11 \mathrm{ug} / \mathrm{dL}$ (o $300 \mathrm{nmol} / \mathrm{L}$ ) en fase aguda han mostrado asociación con desenlaces clínicos desfavorables ${ }^{6,15}$.

\section{b) Hipotiroidismo central versus síndrome de enfermedad no tiroidea (NTIS)}

Múltiples estudios han evidenciado que los pacientes graves presentan alteraciones funcionales del eje tirotropo descritos como NTIS (previamente conocido como síndrome del eutiroideo enfermo). Según la gravedad del paciente, existe un continuo desde hallazgos de T3 o T3 libre bajos aislados, a T3 y T4 total o libre bajos con una respuesta hipofisaria inapropiada con niveles normales o disminuidos de $\mathrm{TSH}^{26}$. Este continuo se correlaciona con la gravedad del compromiso sistémico y riesgo de muerte ${ }^{27}$, y reflejaría una respuesta adaptativa para disminuir el metabolismo, que evoluciona con normalización espontánea de las alteraciones tiroideas tras la mejoría clínica ${ }^{27}$. El problema se suscita dado que el patrón es el mismo que se observa en el hipotiroidismo central, y la sobresuplementación en NTIS podría ser perjudicial para el paciente ${ }^{26,27}$. Debido a la mayor vida media de las hormonas tiroideas y la concurrencia frecuente con NTIS, es que no se sugiere una evaluación de eje tiroideo en agudo, sino solo ante hallazgos clínicos sugerentes como bradicardia, hipotermia, miopatía, deterioro cognitivo o hiponatremia persistente.

\section{c) Compromiso neurohipofisario}

Respecto a los trastornos de la hormona antidiurética $(\mathrm{ADH})$, el aporte de altos volúmenes de cristaloides en la reanimación inicial, la disfunción renal frecuente asociada al politrauma y el uso de suero hipertónico en pacientes neurocríticos pueden favorecer estados poliúricos asociados a hipernatremia, lo que puede dificultar el diagnóstico diferencial con DI. En estos casos, la osmolalidad urinaria (OsmU), o más indirectamente la densidad urinaria, asociada a la medición de sodio urinario $(\mathrm{NaU})$ en muestra aislada, son de gran ayuda. Se confirma una DI al objetivar hipernatremia asociada a poliuria hipotónica con la presencia de OsmU inapropiadamente normal o baja $(<300 \mathrm{mOsm} / \mathrm{Kg})$ para una hiperosmolalidad plasmática, además de un $\mathrm{NaU}$ habitualmente bajo por su dilución en agua libre. El hallazgo de hipernatremia asociada a orina no hipoosmolar o un $\mathrm{NaU}$ elevado sugiere poliuria por sobrecarga salina.

Por otra parte, el desarrollo de hiponatremia también es frecuente en los pacientes con TEC en contexto de politrauma, por activación no osmolar de la síntesis y secreción de ADH ante la hipotensión o hipovolemia, hipocortisolismo o hipotiroidismo agudos, o secreción inapropiada por lesión traumática cerebral o por fármacos. El principal diagnóstico diferencial de hiponatremia en pacientes con TEC es la encefalopatía perdedora de sal. En esta última, la evaluación clínica de la presión de perfusión y volemia estimadas, junto a la medición de la OsmU y NaU y la ausencia de condiciones como hipocortisolismo e hipotiroidismo permiten esclarecer el diagnóstico. 
Dado que la disnatremia por trastornos por exceso o deficiencia de ADH se asocian a mayor morbimortalidad, la gran mayoría de los grupos de expertos recomiendan su evaluación rutinaria ${ }^{28}$.

\section{d) Otros ejes}

$\mathrm{Al}$ igual que los ejes corticotropo y tirotropo, los pacientes graves producto de un politraumatismo presentan habitualmente una inhibición adaptativa del eje gonadotropo y somatotropo e hiperprolactinemia hasta en $70 \%$ de los casos, que no se asocian a una mayor mortalidad y no cambian conducta terapéutica, por lo que existe consenso en que no es costo-efectiva su evaluación en $\operatorname{agudo}^{29}$.

\section{Fase crónica}

Los estudios prospectivos que han evaluado la función hipofisaria son consistentes en revelar una tasa significativa de compromiso de novo y también la recuperación de ejes alterados en agudo, por lo que se recomienda la evaluación de todos los ejes en fase crónica ${ }^{6}$. Tanriverdi y colaboradores evaluaron de forma prospectiva los ejes hipofisarios en la fase aguda y crónica (12 meses post-TEC), encontrando $19,2 \%$ de hipocortisolismo y $51,9 \%$ de déficits de novo en uno o más ejes en fase crónica ${ }^{30}$. Existe una alta variabilidad de prevalencia entre los estudios,con respecto a la deficiencia de hormona de crecimiento $(\mathrm{GH})$, descrita entre 9 y $63 \%$ en fase crónica ${ }^{31}$. Esto puede explicarse por diferencias en la temporalidad de la medición y en el tipo de test de estímulo de $\mathrm{GH}$ aplicados ${ }^{31}$. Si bien en pacientes con $\geq 3$ ejes comprometidos basta una medición de IGF-1 bajo el rango normal para diagnosticar insuficiencia, muchos de los pacientes con HTEC pueden presentar déficit aislado y requerir un test de estimulación ${ }^{31,32}$. El único test disponible en Chile con alto rendimiento diagnóstico es el test de tolerancia insulínica, pero está contraindicado en pacientes con riesgo convulsivo. Otros, como el de arginina-GHRH, macimorelina y de glucagón aún no se realizan en nuestro país, pero son seguros y serían una buena alternativa.

\section{Diagnóstico diferencial del HTEC}

Considerando que el HTEC es una entidad frecuente y con morbimortalidad asociada, es de suma importancia el diagnóstico diferencial con el síndrome de estrés postraumático post-TEC o síndrome postconmoción cerebral, definido como un conjunto de síntomas somáticos, cognitivos, emocionales y del comportamiento que aparecen luego de un $\mathrm{TEC}^{33}$. Este cuadro puede compartir muchos síntomas con HTEC, como las alteraciones de la concentración y memoria, síntomas depresivos, disminución de la libido y rendimiento sexual, fatiga crónica, entre otros, lo que puede llevar a confusión diagnóstica, subdiagnóstico y a un manejo inadecuado ${ }^{34}$.

\section{Algoritmo de evaluación y abordaje terapéutico del HTEC}

Diversos autores y sociedades han propuesto guías para el manejo del HTEC que tienen algunas diferencias menores, pero con el objetivo común de orientación diagnóstica y de manejo de esta entidad aún poco sospechada ${ }^{1,6,17,35-37}$.

Con la evidencia disponible hasta ahora, sugerimos que todo paciente con TEC moderado a grave sea evaluado en la fase aguda con natremia, diuresis y cortisol sérico matinal. Este último debe ser medido en ausencia del uso de cualquier formulación de glucocorticoides (ej. dexametasona), con el fin de identificar precozmente la presencia de insuficiencia corticotropa y DI que corresponden a las principales causas de morbimortalidad en agudo (Figura 3 ).

Basándonos en los distintos estudios publicados y por un margen de seguridad, proponemos que un cortisol sérico $<11 \mathrm{ug} / \mathrm{dL}$ sea considerado insuficiente y sugerimos iniciar hidrocortisona en dosis fisiológicas o de estrés, dependiendo de la condición clínica del paciente. Valores $\geq 15 \mathrm{ug} /$ $\mathrm{dL}$ pueden ser considerados como suficientes $\mathrm{y}$ valores intermedios (entre 11 y 14,9 ug/dL) deben reevaluarse durante el período agudo y subagudo tanto clínica como bioquímicamente. En caso de no disponer de cortisol sérico o ante valores intermedios persistentes, sugerimos iniciar hidrocortisona empírica, si hay sospecha clínica de insuficiencia corticotropa, como la presencia de hipotensión arterial sostenida o dependencia de drogas vasoactivas para mantener una adecuada presión arterial, hipoglicemia sin causa evidente, hiponatremia persistente, entre otros.

En la fase crónica, ya que la disfunción puede 


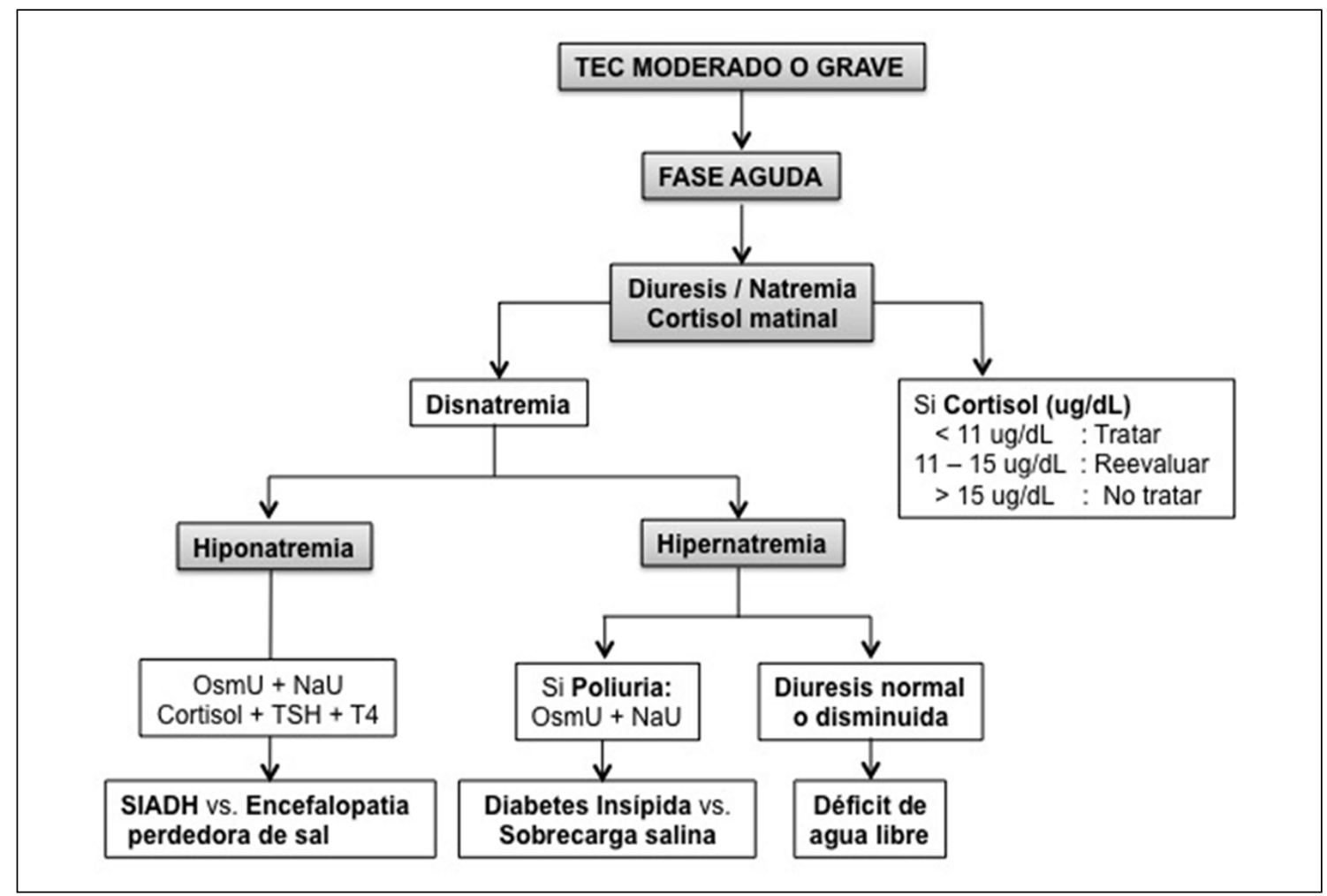

Figura 3. Algoritmo de estudio de HTEC en fase aguda.

aparecer de novo en forma diferida hasta en $51,9 \%$ de los pacientes ${ }^{30}$, independiente de si hubo hipopituitarismo en la fase aguda, proponemos que todo paciente sea reevaluado entre los 3-6 meses y luego un año posterior al trauma, con estudio de todos los ejes hipofisarios: cortisol sérico o basal y post ACTH, TSH + T4 total o libre, prolactina, IGF-1 y FSH + LH + testosterona total en hombres, FSH + estradiol en mujeres en edad fértil con oligo/amenorrea o solo FSH en postmenopáusicas. En caso de sospecharse o confirmarse un HTEC, derivar a endocrinología para tratamiento (Figura 4).

Como ejemplo, exponemos el caso de un hombre de 22 años previamente sano, que presentó un accidente de tránsito de alta energía en motocicleta, con TEC grave en GCS 7, con fractura expuesta de cráneo y macizo facial, pérdida de masa encefálica, extenso compromiso contuso hemorrágico frontal y de quiasma óptico y daño axonal difuso grado II con necesidad de tratamiento quirúrgico; además de exposición corneal con

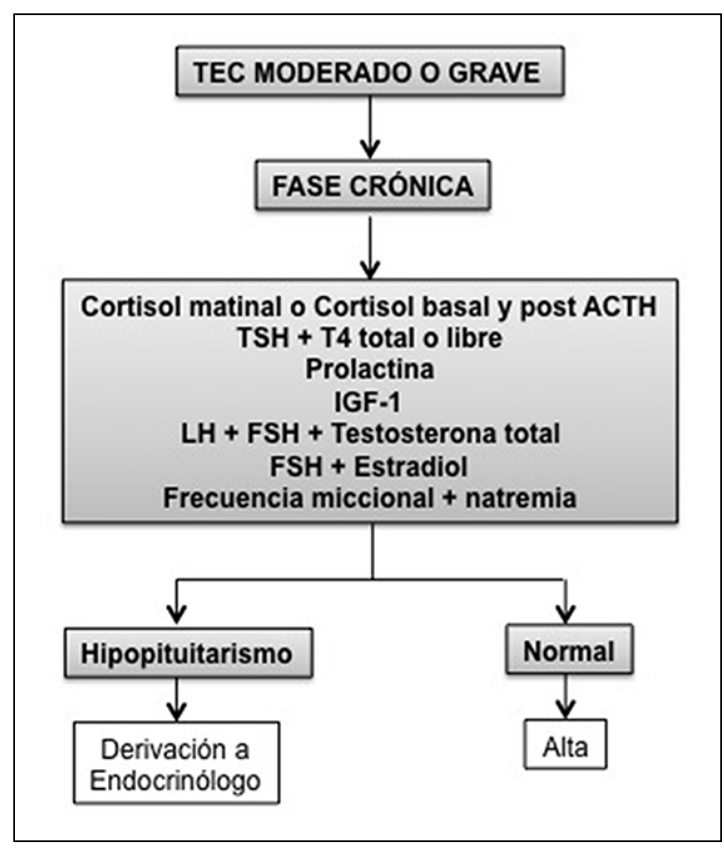

Figura 4. Algoritmo de estudio de HTEC en fase crónica. 


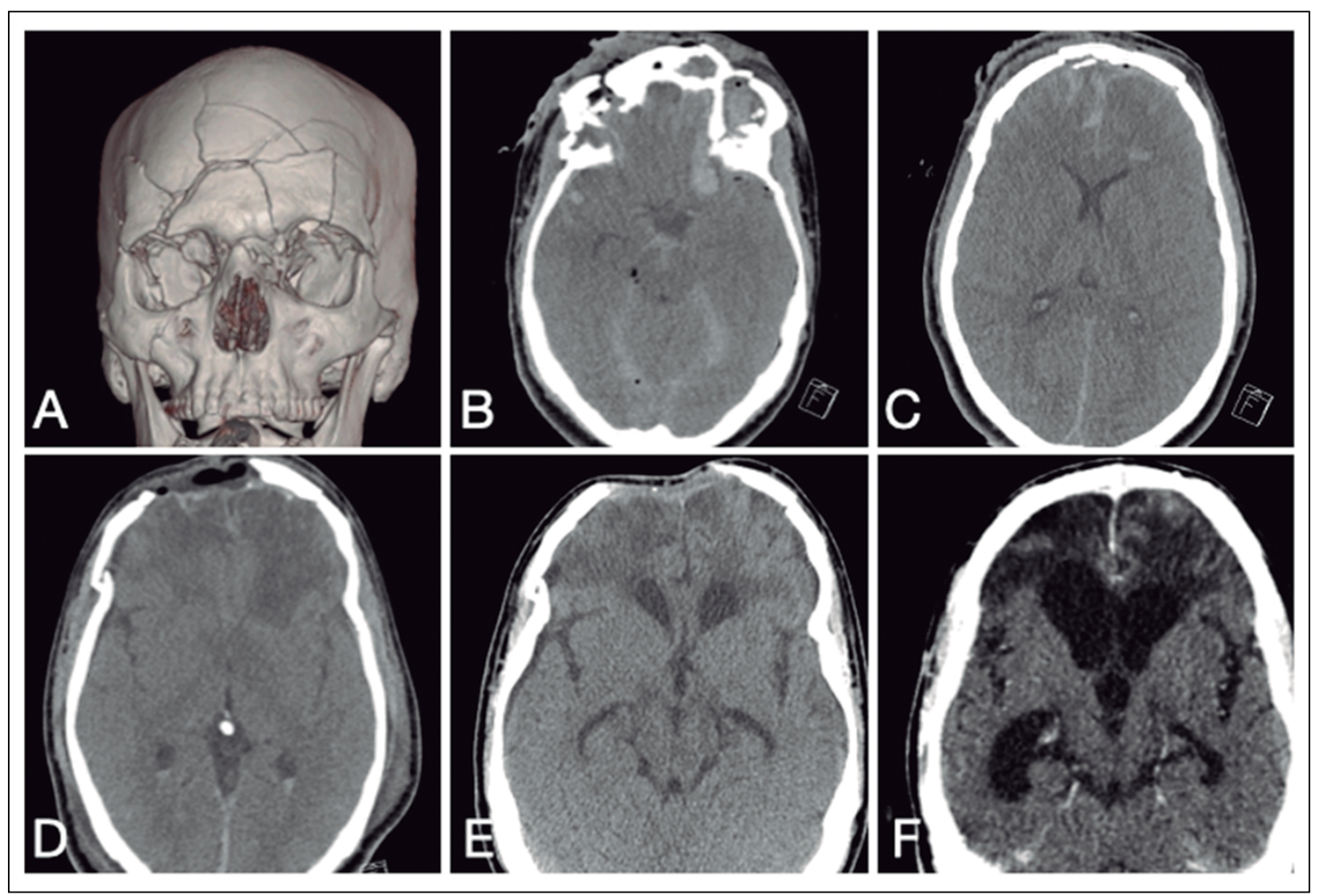

Figura 5. Tomografías computadas (TC) de cerebro al diagnóstico y en el seguimiento del caso clínico expuesto. (A) Reformateo 3D VR de TC de cráneo que muestra fractura facial orbitofrontal compleja LeFort tipo III. (B, C) Cortes axiales de TC de encéfalo sin contraste que muestra hemorragia subaracnoidea traumática, hematoma subdural laminar tentorial y contusiones cerebrales bifrontales. TC de encélalo de seguimiento (D) 4 días después, (E) un mes después y (F) un año después del trauma muestra la evolución de las contusiones frontales con atrofia progresiva del encéfalo y dilatación ex vacuo de los ventrículos.

proptosis a izquierda y múltiples fracturas (Figura $5 \mathrm{~A}-\mathrm{E}$ ). A las $48 \mathrm{~h}$ de su ingreso, evolucionó con poliuria e hipernatremia compatibles con DI, con requerimientos de desmopresina, sin hallazgos específicos en imágenes selares (Figura 6A).

A los 6 días del accidente se trasladó a nuestro centro, donde persistió con requerimientos de desmopresina, sin hipocortisolismo, por lo que se mantuvo el tratamiento inicial (Tabla 2).

$\mathrm{Al}$ mes de evolución, se encontraba clínicamente estable, ya extubado y sin uso de vasoactivos, neurológicamente vigil y obedeciendo órdenes simples, sin episodios convulsivos bajo tratamiento anticonvulsivante, pero con requerimientos de opioides, antidepresivos y neurolépticos para manejo conductual. Además, presentaba deterioro musculoesquelético significativo por reposo prolongado, pese a terapia de rehabilitación kinésica y soporte nutricional. En este contexto se decidió reevaluar precozmente la función hipofisaria, pesquisándose cortisol sérico indeterminado $(9,1 \mathrm{ug} /$ $\mathrm{dL})$, por lo que se inició sustitución con hidrocortisona, y valores de testosterona total $(102 \mathrm{ng} / \mathrm{dL})$ y libre calculada disminuidas. Dado el contexto del paciente, se decidió suplementar con testosterona micronizada transdérmica (TTD) $25 \mathrm{mg} /$ día. Se objetivó persistencia de DI adíptica, por lo que se tituló aporte de agua libre y desmopresina para diuresis y natremia normales.

A 6 meses del TEC, la reevaluación de la función hipofisaria mostró persistencia de DI adíptica, donde la OsmU adecuada (757 mOsm/ $\mathrm{kg}$ ) ayudó a discernir que su hipernatremia persistente correspondía a falta de ingesta hídrica y no a dosis insuficiente de desmopresina. También se objetivó un aumento de testosterona total 


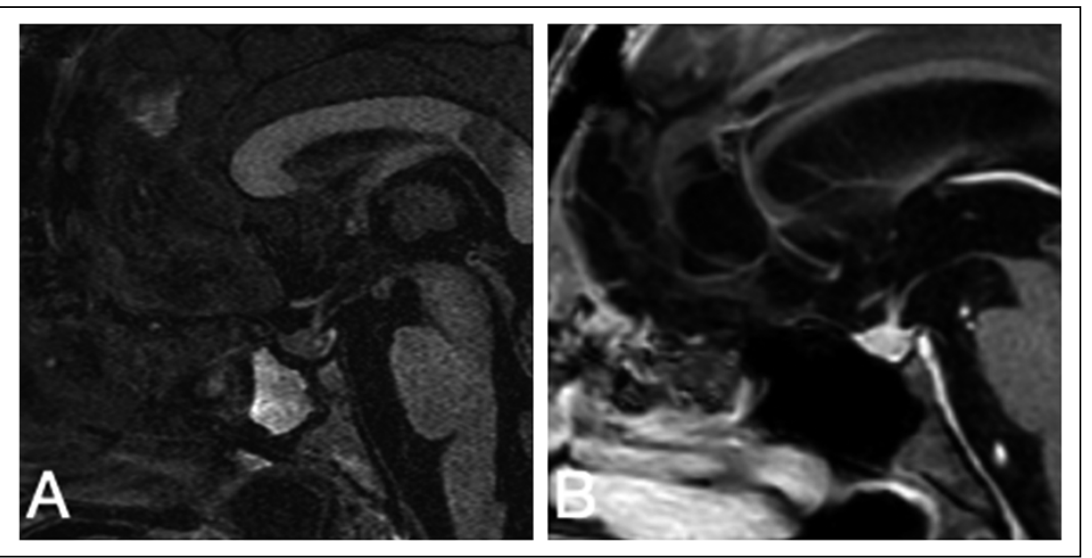

Figura 6. Imágenes selares por resonancia magnética del caso clínico expuesto. Cortes sagitales en línea media de encéfalo, ponderados en T1 y centrados en región selar (A) sin contraste 5 días después del trauma y (B) con contraste 2 años después del trauma. Se demuestra un volumen normal de la adenohipófisis, sin atrofia progresiva, sin evidencias de sección macroscópica de tallo hipofisiario.

a rango subóptimo ( $240 \mathrm{ng} / \mathrm{dL})$, por lo que se suspendió el uso de TTD. La evaluación de la función corticotropa mostró valores indeterminados de cortisol $(13,6 \mathrm{ug} / \mathrm{dL})$, por lo que se mantuvo con hidrocortisona, dado imposibilidad de realizar test de estimulación con ACTH en ese momento. Por otra parte, se evidenció la aparición de compromiso tirotropo sintomático tras el hallazgo bioquímico de niveles de T4 libre bajos, TSH normal y síntomas depresivos que mejoraron con levotiroxina 75 ug al día.

$\mathrm{Al}$ año post-TEC, la evaluación endocrina mostró persistencia del compromiso tirotropo y DI, recuperación del eje corticotropo y gonadotropo,

Tabla 2. Evaluación hormonal del caso clínico en fase aguda, subaguda y crónica

\begin{tabular}{|c|c|c|c|c|}
\hline Examen & $\begin{array}{c}6^{\circ} \text { día } \\
\text { postrauma }\end{array}$ & $\begin{array}{c}1 \text { mes } \\
\text { postrauma }\end{array}$ & $\begin{array}{c}6 \text { meses } \\
\text { postrauma }\end{array}$ & 1 año \\
\hline (135-145 mEq/L) & 162 & $\begin{array}{c}153 \\
\text { (con DDAVP) }\end{array}$ & $\begin{array}{c}149 \\
\text { (con DDAVP) }\end{array}$ & $\begin{array}{c}147 \\
\text { (con DDAVP) }\end{array}$ \\
\hline$(40-220 \mathrm{mEq} / \mathrm{L})$ & $<20$ & --- & 150 & \\
\hline$(275-295 \mathrm{mOsm} / \mathrm{k})$ & --- & --- & 311 & \\
\hline Variable (mOsm/k) & --- & --- & 757 & \\
\hline Cortisol sérico AM & 12,6 & 9,1 & 13,6 & 17,6 \\
\hline Cortisol post ACTH & --- & --- & --- & 26,8 \\
\hline$(100-367$ ng/mL) & 401 & --- & 307 & 260 \\
\hline$(1,7-8,6 \mathrm{mUl} / \mathrm{mL})$ & --- & 2,2 & 3,2 & 5,0 \\
\hline Testosterona total $\quad(249-836 \mathrm{ng} / \mathrm{dL})$ & --- & 102 & 240 (1 día \$ TD) & $339(\sin T D)$ \\
\hline$(16,5-55 \mathrm{nmol} / \mathrm{L})$ & --- & 14,5 & --- & 9,09 \\
\hline$(0,3-4,2 \mathrm{uUl} / \mathrm{mL})$ & 1,89 & 1,92 & 1,89 & 2,13 \\
\hline$(4,6-12 u g / d L)$ & 5,9 & --- & --- & 6,3 (75 ug) \\
\hline$(0,93-1,7 \mathrm{ng} / \mathrm{dL})$ & --- & 1,26 & 0,68 & --- \\
\hline (4-15 ng/mL) & 30,3 & --- & 41,1 & 6,9 \\
\hline
\end{tabular}


y persistencia de IGF-1 normal sobre percentil 50, sin síntomas de deficiencia de $\mathrm{GH}$ por lo que no se realizó test de estimulación de hormona de crecimiento (Tabla 2). Las Figuras 5E-F y 6B muestran la evolución imagenológica en este caso.

Aplicando el algoritmo de aproximación, este permitió el diagnóstico y tratamiento en la fase aguda del compromiso neurohipofisario. De manera excepcional, se midió prolactina como elemento predictor de hipopituitarismo ante valores disminuidos, y el eje tirotropo para descartar un compromiso grave que ameritara corrección. Con el objetivo de optimizar la recuperación del paciente dado el deterioro cognitivo y musculoesquelético evidenciado, y considerando que se contaba con un endocrinólogo experimentado para la interpretación de los resultados, se reevaluó la función hipofisaria precozmente al mes. Así, se detectó un probable hipocortisolismo e hipogonadismo, sugiriéndose el aporte de TTD dado el deterioro musculoesquelético grave que presentaba. Posteriormente, la reevaluación en fase crónica permitió la pesquisa del compromiso tirotropo de novo que pudo perjudicar su recuperación, $\mathrm{y}$ se objetivó la recuperación del eje corticotropo y gonadotropo, evitando una suplementación permanente innecesaria.

\section{Conclusiones}

El HTEC es un trastorno frecuentemente subdiagnosticado y subtratado, con importante morbimortalidad asociada. Una mayor difusión de esta condición permitirá aumentar su identificación precoz por los equipos de unidades de paciente crítico. El abordaje protocolizado de esta condición, tanto en fase aguda como crónica, impacta favorablemente en el pronóstico de estos pacientes.

Dado que en fase aguda la principal dificultad radica en la interpretación de los ejes hipofisarios, proponemos un algoritmo simple de estudio y manejo del eje corticotropo y disnatremia para que todo equipo a cargo de estos pacientes pueda aplicarlo, y solicitar apoyo del endocrinólogo ante el desarrollo de DI o duda diagnóstica.

En fase crónica, el principal diagnóstico diferencial es el síndrome de estrés postconmoción cerebral. Dada la alta prevalencia de déficits hipofisarios de novo y la posibilidad de recuperar una deficiencia previa adquirida en fase aguda, recomendamos la reevaluación de todos los ejes hipofisarios entre los 3-6 meses y luego a los 12 meses post-TEC, y derivar al endocrinólogo para su terapia.

\section{Referencias}

1. Quinn M, Agha A. Post-Traumatic Hypopituitarism-Who Should Be Screened, When, and How? Front Endocrinol (Lausanne). 2018; 9: 8.

2. Management of Concussion/mTBI Working Group. VA/DoD Clinical Practice Guideline for Management of Concussion/Mild Traumatic Brain Injury. J Rehabil Res Dev 2009; 46 (6): CP1-68.

3. Schneider HJ, Kreitschmann-Andermahr I, Ghigo E, Stalla GK, Agha A. Hypothalamopituitary dysfunction following traumatic brain injury and aneurysmal subarachnoid hemorrhage: a systematic review. JAMA 2007; 298 (12): 1429-38.

4. Cyran E. Hypophysenschädigung durch Schädelbasisfraktur. Dtsch Med Wochenschr 1918; 44 (45).

5. Keynes M. The medical health of Napoleon Bonaparte. J Med Biogr 1996; 4 (2): 108-17.

6. Tanriverdi F, Kelestimur F. Pituitary dysfunction following traumatic brain injury: clinical perspectives. Neuropsychiatr Dis Treat 2015; 11: 1835-43.

7. Dusick JR, Wang C, Cohan P, Swerdloff R, Kelly DF. Pathophysiology of hypopituitarism in the setting of brain injury. Pituitary 2012; 15 (1): 2-9.

8. Bondanelli M, Ambrosio MR, Carli A, Bergonzoni A, Bertocchi A, Zatelli MC, et al. Predictors of pituitary dysfunction in patients surviving ischemic stroke. J Clin Endocrinol Metab 2010; 95 (10): 4660-8.

9. Daniel PM, Prichard MM, Treip CS. Traumatic infarction of the anterior lobe of the pituitary gland. Lancet (London, England) 1959; 2 (7109): 927-31.

10. Kornblum RN, Fisher RS. Pituitary lesions in craniocerebral injuries. Arch Pathol 1969; 88 (3): 242-8.

11. Salehi F, Kovacs K, Scheithauer BW, Pfeifer EA, Cusimano M. Histologic study of the human pituitary gland in acute traumatic brain injury. Brain Inj 2007; 21 (6): 651-6.

12. Wachter D, Gündling K, Oertel MF, Stracke H, Böker DK. Pituitary insufficiency after traumatic brain injury. J Clin Neurosci 2009; 16 (2): 202-8.

13. Tanriverdi F, De Bellis A, Bizzarro A, Sinisi AA, Bellastella G, Pane E, et al. Antipituitary antibodies after traumatic brain injury: is head trauma-induced pituitary dysfunction associated with autoimmunity? Eur J Endocrinol 2008; 159 (1): 7-13. 
14. Tanriverdi F, De Bellis A, Ulutabanca H, Bizzarro A, Sinisi AA, Bellastella G, et al. A five year prospective investigation of anterior pituitary function after traumatic brain injury: is hypopituitarism long-term after head trauma associated with autoimmunity? J Neurotrauma 2013; 30 (16): 1426-33.

15. Hannon MJ, Sherlock M, Thompson CJ. Pituitary dysfunction following traumatic brain injury or subarachnoid haemorrhage - in "Endocrine Management in the Intensive Care Unit". Best Pract Res Clin Endocrinol Metab 2011; 25 (5): 783-98.

16. Klose M, Feldt-Rasmussen U. Chronic endocrine consequences of traumatic brain injury - what is the evidence? Nat Rev Endocrinol 2018; 14 (1): 57-62.

17. Tritos NA, Yuen KC, Kelly DF; AACE Neuroendocrine and Pituitary Scientific Committee. American Association of Clinical Endocrinologists and American College of Endocrinology Disease State clinical review: a neuroendocrine approach to patients with traumatic brain injury. Endocr Pract 2015; 21 (7): 823-31.

18. Tanriverdi F, Unluhizarci K, Kelestimur F, Sherlock M, Cooper MS. Endocrinology of acute brain injury. Godoy DA, editor. Torino, Italy: SEEd Medical Publishers; 2013.

19. Tanriverdi F, Ulutabanca H, Unluhizarci K, Selcuklu A, Casanueva FF, Kelestimur F. Pituitary functions in the acute phase of traumatic brain injury: are they related to severity of the injury or mortality? Brain Inj 2007; 21 (4): 433-9.

20. Téblick A, Langouche L, Van den Berghe G. Anterior pituitary function in critical illness. Endocr Connect 2019; 8 (8): R131-r43.

21. Cooper MS, Stewart PM. Corticosteroid insufficiency in acutely ill patients. N Engl J Med 2003; 348 (8): 727-34.

22. Pizarro CF, Troster EJ. Adrenal function in sepsis and septic shock. J Pediatr 2007; 83 (5 Suppl): S155-62.

23. Mason PE, Al-Khafaji A, Milbrandt EB, Suffoletto BP, Huang DT. CORTICUS: the end of unconditional love for steroid use? Crit Care 2009; 13 (4): 309.

24. Cohan P, Wang C, McArthur DL, Cook SW, Dusick JR, Armin B, et al. Acute secondary adrenal insufficiency after traumatic brain injury: a prospective study. Crit Care Med 2005; 33 (10): 2358-66.

25. Marik PE, Pastores SM, Annane D, Meduri GU, Sprung $\mathrm{CL}$, Arlt W, et al. Recommendations for the diagnosis and management of corticosteroid insufficiency in critically ill adult patients: consensus statements from an international task force by the American College of Critical Care Medicine. Crit Care Med 2008; 36 (6):
1937-49.

26. Wang YF, Heng JF, Yan J, Dong L. Relationship between disease severity and thyroid function in Chinese patients with euthyroid sick syndrome. Medicine 2018; 97 (31): e11756.

27. Gutch M, Kumar S, Gupta KK. Prognostic Value of Thyroid Profile in Critical Care Condition. Indian J Endocrinol Metab 2018; 22 (3): 387-91.

28. Hannon MJ, Crowley RK, Behan LA, O'Sullivan EP, O'Brien MM, Sherlock M, et al. Acute glucocorticoid deficiency and diabetes insipidus are common after acute traumatic brain injury and predict mortality. J Clin Endocrinol Metab 2013; 98 (8): 3229-37.

29. Scranton RA, Baskin DS. Impaired Pituitary Axes Following Traumatic Brain Injury. J Clin Med 2015; 4 (7): 1463-79.

30. Tanriverdi F, Senyurek H, Unluhizarci K, Selcuklu A, Casanueva FF, Kelestimur F. High risk of hypopituitarism after traumatic brain injury: a prospective investigation of anterior pituitary function in the acute phase and 12 months after trauma. J Clin Endocrinol Metab 2006; 91 (6): 2105-11.

31. Kgosidialwa O, Hakami O, Muhammad Zia-Ul-Hussnain H, Agha A. Growth Hormone Deficiency Following Traumatic Brain Injury. Int J Mol Sci 2019; 20 (13).

32. Yuen KCJ, Biller BMK, Radovick S, Carmichael JD, Jasim S, Pantalone KM, et al. American Association of Clinical Endocrinologists and American College of Endocrinology Guidelines for management of growth hormone deficiency in adults and patients transitioning from pediatric to adult care. Endocr Pract 2019; 25 (11): 1191-232.

33. American Psychiatric Association. Neurocognitive Disorders. En: Diagnostic and Statistical Manual of Mental Disorders. Amberton University. e-Course Syllabus. 2020.

34. Sharp DJ, Jenkins PO. Concussion is confusing us all. Prac Neurol 2015; 15 (3): 172-86.

35. Fernández-Rodríguez E, Bernabeu I, Castro AI, Casanueva FF. Hypopituitarism after traumatic brain injury. Endocrinol Metab Clin North Am 2015; 44 (1): 151-9.

36. Tan CL, Alavi SA, Baldeweg SE, Belli A, Carson A, Feeney $C$, et al. The screening and management of pituitary dysfunction following traumatic brain injury in adults: British Neurotrauma Group guidance. J Neurol Neurosurg Psychiatry 2017; 88 (11): 971-81.

37. Tan CL, Hutchinson PJ. A neurosurgical approach to traumatic brain injury and post-traumatic hypopituitarism. Pituitary 2019; 22 (3): 332-7. 